\title{
AGREGACIÓN Y CAMBIO EN TASAS LINEALES: TASAS DE PARTICIPACIÓN FAMILIAR EN LA ACTIVIDAD ECONÓMICA
}

\author{
FERNANDO CORTÉS Y ROSA MA. RUBALCAVA \\ El Colegio de México
}

\section{Introducción}

EL TEMA QUE SE DESARROLla en este trabajo surgió como un intento de respuesta a inquietudes planteadas en la investigación de los determinantes de las tasas de participación familiar en el mercado de trabajo.* El interés residía en el examen del impacto que tienen sobre la tasa globa! de participación los grupos y subgrupos que se construyen a partir de unidades familiares.

La dinámica de la indagación llevó a incluir además del problema de la agregación, el cstudio del cambio en el tiempo. Esta preocupación no formaba parte de la demanda original.

La aplicación del modelo de análisis que proponemos supera el ámbito de la composición y cambio de la participación familiar en la actividad productiva. En efecto, como lo mostrarán las ecuaciones de la sección II, los resultados son válidos para cualquier indicador que pueda expresarse como promedio ponderado.

Sin embargo, en la sección III se verá, a través de un ejemplo hipotético tomado de la inserción laboral de los miembros de un conjunto de familias, el manejo operativo de las ecuaciones que constituyen e] modelo.

Como la demanda proviene de un estudio centrado en las mediciones referidas a un grupo dado de observaciones en un punto del tiempo, han quedado fuera de los límites de este trabajo las consideraciones relativas a las variaciones que afectan a la cantidad de unidades a través del tiempo. Este tópico requiere de un tratamiento especial y tendrá que ser objeto de futuras investigaciones.

* Brígida García, Humberto Muñoz y Orlandina de Oliveira, Hogares y trabajadores en la Ciudad de México, 1978. 


\section{Presentación del modelo}

Supóngase que el valor de un indicador global y puede expresarse como una combinación de $\underline{\text { n }}$ elementos:

$$
\begin{aligned}
& Y=x_{1} p_{1}+x_{2} P_{2}+\ldots \ldots \ldots \ldots+x_{n} p_{n} \\
& Y=\sum_{j=1}^{n} x_{i} P_{i}
\end{aligned}
$$

con la condición de que:

$$
\underset{j=1}{n} p_{i}=1
$$

Si con los $n$ componentes decilimos formar $\underline{m}$ grupos de tamaños $n_{1}, n_{2}, \ldots \ldots, n_{m}$; entonces la ecuación (1) se puede reescribir sin pérdida de generalidad de la siguiente manera:

$$
\begin{aligned}
\mathrm{Y} & =\underset{\mathrm{i}=1}{\sum_{1}^{\mathrm{n}_{1}} \mathrm{x}_{1}, \mathrm{i}} \mathrm{P}_{1, \mathrm{i}}+\underset{\mathrm{i}=1}{\mathrm{n}_{2}} \mathrm{x}_{2, \mathrm{i}} \mathrm{P}_{2, \mathrm{i}}+\cdots \cdots+\underset{\mathrm{i}=1}{\mathrm{n}_{\mathrm{m}}} \mathrm{x}_{\mathrm{m}, \mathrm{i}} \mathrm{P}_{\mathrm{m}, \mathrm{i}} \\
& =\underset{\mathrm{h}=1}{\mathrm{~m}} \underset{\mathrm{h}=1}{\mathrm{~m}_{\mathrm{h}} \mathrm{x}_{\mathrm{h}, \mathrm{i}} \mathrm{P}_{\mathrm{h}, \mathrm{i}}}
\end{aligned}
$$

Donde el primer subíndice de $\underline{x}$ y $\underline{p}$ denota el agregado y el segundo simboliza las observaciones.

Los sumandos de las igualdades (1) y (2) pueden interpretarse como las contribuciones que realizan las observaciones individuales y los grupos, respectivamente, a la fórmación del valor de $\mathbf{Y}$. Así por ejemplo, $x_{\mathfrak{i}} p_{j}$ representa el monto con que concurre la i-ésima observación al valor de la combinación. En tanto que:

$$
\underset{j=1}{n_{h}} x_{h i} p_{h i}
$$

mide el aporte del grupo $\underline{h}$.

Para estudiar el cambio de $Y$ a través del tiempo debemos considerar alteraciones tanto en los valores $x$ como en los de $\underline{p}$, los que introducidos en la ecuación (1) generan: 
(3) $\mathrm{Y}+\Delta \mathrm{Y}=\left(\mathrm{x}_{1}+\Delta \mathrm{x}_{1}\right)\left(\mathrm{p}_{1}+\Delta \mathrm{p}_{1}\right)+\left(\mathrm{x}_{2}+\Delta \mathrm{x}_{2}\right)$

$\left(\mathrm{p}_{2}+\Delta \mathrm{p}_{2}\right)+\ldots \ldots \ldots+\left(\mathrm{x}_{\mathrm{n}}+\Delta \mathrm{x}_{\mathrm{n}}\right)\left(\mathrm{p}_{\mathrm{n}}+\Delta \mathrm{p}_{\mathrm{n}}\right)$

en que $\Delta \mathrm{x}_{\mathrm{i}}$ y $\Delta \mathrm{p}_{\mathrm{j}}$ denotan variaciones experimentadas por los componentes de la combinación lineal.

De la diferencia entre (3) y (1) se obtiene:

$$
\begin{array}{r}
\Delta \mathrm{Y}=\left(\mathrm{p}_{1} \Delta \mathrm{x}_{1}+\mathrm{x}_{1} \Delta \mathrm{p}_{1}+\Delta \mathrm{x}_{1} \Delta \mathrm{p}_{1}\right)+\ldots \ldots \ldots \ldots+ \\
\ldots \ldots \ldots+\left(\mathrm{p}_{\mathrm{n}} \Delta \mathrm{x}_{\mathrm{n}}+\mathrm{x}_{\mathrm{n}} \Delta \mathrm{p}_{\mathrm{n}}+\Delta \mathrm{x}_{\mathrm{n}} \Delta \mathrm{p}_{\mathrm{n}}\right)
\end{array}
$$

Los tres términos que encierra cada paréntesis muestran la forma como se descompone la contribución de cada unidad a la variación en $Y$. Examinemos con un poco más de detalle los elementos que conforman el valor con que concurre a la formación de $Y$ la observación genérica $\mathrm{i}$ :

$$
\left(\mathrm{p}_{\mathrm{i}} \Delta \mathrm{x}_{\mathrm{i}}+\mathrm{x}_{\mathrm{i}} \Delta \mathrm{p}_{\mathrm{i}}+\Delta \mathrm{x}_{\mathrm{i}} \Delta \mathrm{p}_{\mathrm{i}}\right)
$$

El término $p_{i} \Delta x_{i}$ tiene una interpretación equivalente a la de derivada parcial ya que indica la parte de la contribución de la i-ésima observación que se origina en un cambio en $x$ manteniendo $p$ constante. El componente $\mathrm{x}_{1} \Delta \mathrm{p}_{1}$. señala aquella parte de la contribución del i-ésimo término que se debe a la variación en $\mathrm{p}$ con $\mathrm{x}$ constante. El tercer componente de la suma $\left(\Delta \mathrm{x}_{\mathrm{j}} \Delta \mathrm{p}_{\mathrm{j}}\right)$ refleja el impacto de las variaciones de $\underline{x}$ y $p$ sobre $\underline{Y}$.

De acuerdo con estas interpretaciones, la variación total que experimenta $\underline{Y}$ resulta de las modificaciones que afectan a cada una de las $n$ unidades. Además, el aporte de las observaciones se puede descomponer en:

(i) El cambio en $\underline{x}$, con $\underline{p}$ fijo.

(ii) El cambio en $\frac{x}{p}$, con $\frac{x}{x}$ fijo.

(iii) La resultante $\overline{\mathrm{de}} \Delta \mathrm{x}$ por $\overline{\Delta p}$.

El mismo tipo de descomposición puede obtenerse para los m grupos. Si para cada uno de los sumandos de (2) se cumple:

$$
\begin{aligned}
& \mathrm{n}_{1} \\
& \Sigma \mathrm{x}_{1}, \mathrm{ip}_{1}, \mathrm{i}=\mathrm{x}_{1} \mathrm{p}_{1} \\
& i=1 \ldots \ldots \ldots \ldots \text {. } \\
& \mathrm{n}_{\mathrm{h}} \ldots \ldots \ldots \ldots \ldots \\
& \Sigma \mathrm{x}_{\mathrm{h}}, \mathrm{i} \mathrm{P}_{\mathrm{n}}, \mathrm{i} \mathrm{Ph}_{\mathrm{i}} \mathrm{i}=\mathrm{x}_{\mathrm{h}} \mathrm{P}_{\mathrm{h}} \\
& \mathrm{i}=1 \ldots \ldots \ldots \ldots . \\
& \mathrm{n}_{\mathrm{m}} \ldots \ldots \ldots \ldots \ldots \\
& \Sigma x_{m}, \mathrm{Pm}, \mathrm{i}=\mathrm{x}_{\mathrm{m}} \mathrm{Pm} \\
& \mathrm{i}=1
\end{aligned}
$$


Donde $\mathrm{X}_{\mathrm{h}}$ y $\mathrm{P}_{\mathrm{h}}$ simbolizan las mediciones calculadas directamente sobre el agregado genérico $\underline{h}$. Si las condiciones representadas por las igualdades (5) son válidas, entonces (2) asume la forma:

$$
\begin{aligned}
Y & =X_{1} P_{1}+X_{2} P_{2}+\ldots \ldots \ldots+X_{m} P_{m} \\
& =\sum_{h=1}^{m} X_{h} P_{h}
\end{aligned}
$$

$$
\operatorname{con} \sum_{h=1}^{m} P_{h}=1
$$

De la solạ observación de la estructura de las ecuaciones (6) y (1) es posible establecer que $\Delta Y$ para los datos agrupados se debe descomponer de la misma forma que (4):

$$
\begin{aligned}
\Delta \mathrm{Y}= & \left(\mathrm{P}_{1} \Delta \mathrm{X}_{1}+\mathrm{X}_{1} \Delta \mathrm{P}_{1}+\Delta \mathrm{X}_{1} \Delta \mathrm{P}_{1}\right)+\ldots \ldots \ldots \\
& \left.\ldots \ldots+\mathrm{P}_{\mathrm{m}} \Delta \mathrm{X}_{\mathrm{m}}+\mathrm{X}_{\mathrm{m}} \Delta \mathrm{P}_{\mathrm{m}}+\Delta \mathrm{X}_{\mathrm{m}} \Delta \mathrm{P}_{\mathrm{m}}\right)
\end{aligned}
$$

Los componentes del término genérico $\underline{h}$ :

$$
\left(\mathrm{P}_{\mathrm{h}} \Delta \mathrm{X}_{\mathrm{h}}+\mathrm{X}_{\mathrm{h}} \Delta \mathrm{P}_{\mathrm{h}}+\Delta \mathrm{X}_{\mathrm{h}} \Delta \mathrm{P}_{\mathrm{h}}\right)
$$

dan cuenta de la contribución del grupo $\underline{h}$ al cambio en el valor de Y. Esta expresión permite, además, individualizar qué parte proviene de la modificación en $x$ manteniendo $p$ constante $\left(P_{h} \Delta X_{h}\right)$, cuál de la alteración en $\underline{P}$ con $\underline{X}$ constante $\left(X_{h} \Delta P_{h}\right)$ y aquella que se origina en las variaciones simultáneas en $\underline{X}$ y $\underline{P}\left(\Delta X_{h} \Delta P_{h}\right)$.

Las ecuaciones (5) vinculan las cuotas de los grupos y de las observaciones en lo que tiene que ver con la determinación de los valores de $Y$ y $\Delta Y$. Nos dicen que $X_{h} P_{h}$ es igual a la suma de las contribuciones correspondientes a las unidades que conforman el agregado $\underline{h}$.

La información referida a los aportes de los grupos a la combinación lineal y a su cambio, expresada por (6) y (7), puede complementarse con las ecuaciones (5) para recuperar los componentes individuales.

En resumen, este conjunto de ecuaciones muestran la composición de $\underline{Y}$ y $\Delta Y$, en términos de los impactos agregados e individuales. Además, nos señalan el tipo de relaciones que establecen vínculos entre los grupos y las unidades con que se integran. 


\section{Tasas de participación familiar: un ejemplo}

En esta sección ilustraremos los caminos analíticos que se abren con el modelo que presentamos en el apartado anterior. Mostraremos a través de un ejemplo numéricu sencillo la manera como se aplican las ecuaciones de descomposición y cambio, a las tasas de participación de las familias en el mercado de trabajo.

Definamos la tasa global de participación laboral $(\underline{Y})$ como la relación entre el número de personas de doce o más años que trabajan y el total de sujetos de esas edades. Sea la tasa de participación de la familia genérica $\underline{i}\left(x_{j}\right)$ el cociente del número de miembros que trabajan y que tienen doce o más años de edad y el total de miembros de esa familia que cumplen con el mismo requisito de edad. Considérese, por último, que $p_{i}$ denote la importancia relativa de la familia $i$ en la población económicamente activa global: resulta de dividir el número de miembros de doce años o más de la familia entre el total de económicamente activos en la población.

Este conjunto de definiciones cumple con los requisitos que exige la ecuación (1):

$$
\begin{aligned}
& \frac{T R A B \geqslant 12}{\text { P.E.A. }}=\frac{(T R A B \geqslant 12)_{1}}{(\text { P.E.A. })_{1}} \cdot \frac{(\text { P.E.A. })_{1}}{\text { P.E.A. }}+\frac{(\text { TRAB } \geqslant 12)_{2}}{(\text { P.E.A. })_{2}} \cdot \frac{(\text { P.E.A. })_{2}}{\text { P.E.A. }} \\
& +\ldots \ldots \ldots+(\text { TRAB } \geqslant 12)_{n} \cdot(\text { P.E.A. })_{n} \\
& \text { (P.E.A.)n P.E.A. }
\end{aligned}
$$

y además:

$$
-\frac{1}{\text { P.E.A. }} \underset{\mathrm{i}=1}{\mathrm{n}}(\text { P.E.A. })^{1}=1
$$

(TRAB. $\geqslant 12$ ) representa a los individuos de 12 o más años que trajan en la población total. Cuando a dicha expresión se le adscribe un subíndice, se refiere al mismo concepto, pero esta vez aplicado a la familia que individualiza dicho subíndice. La simbología referida a la población económicamente activa es equivalente: P.E.A. denota a la global, y la que caracteriza a la famila $\underline{i}$ se simboliza como (P.E.A.) ${ }_{1}$.

Para examinar el uso de las ecuaciones, consideremos el siguiente ejemplo numérico en que suponemos que se dispone de información para cuatro familias observadas a cinco años de distancia. 
Tabla $\mathrm{N}^{\circ} 1$

Distribucion por edades y situación laboral de cuatro familias observadas en dos instantes $\#$

\begin{tabular}{|c|c|c|c|c|c|c|c|c|c|c|c|c|c|c|c|c|}
\hline \multirow{3}{*}{$\begin{array}{l}\text { Rel. de } \\
\text { parent. }\end{array}$} & \multicolumn{8}{|c|}{ Tiempo 1} & \multicolumn{8}{|c|}{ Tiempo 2} \\
\hline & \multicolumn{2}{|c|}{$F_{1}$} & \multicolumn{2}{|c|}{$\mathrm{F}_{2}$} & \multicolumn{2}{|c|}{$\bar{F}_{3}$} & \multicolumn{2}{|c|}{$\mathrm{F}_{4}$} & \multicolumn{2}{|c|}{$F_{1}$} & \multicolumn{2}{|c|}{$F_{2}$} & \multicolumn{2}{|c|}{$\overline{\mathbf{F}}_{3}$} & \multicolumn{2}{|c|}{$\mathrm{F}_{4}$} \\
\hline & $\mathrm{E}$ & $\mathrm{T}$ & E & $T$ & $E$ & $T$ & $\mathrm{E}$ & $\mathrm{T}$ & $\mathbf{E}$ & $\mathbf{T}$ & $\mathrm{E}$ & $\mathbf{T}$ & $E$ & $\mathrm{~T}$ & $E$ & $\mathrm{~T}$ \\
\hline$P$ & 39 & Si & 45 & Si & 30 & si & 60 & si & 44 & Si & 50 & Si & 35 & si & - & - \\
\hline M & 36 & si & 40 & No & 28 & No & 55 & No & 41 & Si & 45 & Si & 33 & si & 60 & Si \\
\hline $\mathrm{H}_{1}$ & 13 & No & 21 & Si & 12 & Si & 30 & Si & 18 & No & 26 & Si & 17 & si & 35 & $S_{i}$ \\
\hline $\mathrm{H}_{2}$ & 12 & No & 18 & No & 10 & No & 28 & $\mathbf{S i}$ & 17 & No & 23 & No & 15 & Si & 33 & $S_{i}$ \\
\hline $\mathrm{H}_{3}$ & 8 & No & - & - & 9 & No & 27 & $\mathbf{S}_{1}$ & 13 & No & - & - & 14 & No & 32 & Si \\
\hline $\mathrm{H}_{4}$ & - & - & - & - & 8 & No & 26 & Si & 4 & No & - & - & 13 & No & 31 & Si \\
\hline $\mathrm{H}_{5}$ & - & - & - & - & - & - & 23 & si & - & - & - & - & - & - & 28 & Si \\
\hline $\mathrm{H}_{6}$ & - & - & - & - & - & - & 18 & No & - & - & - & - & - & - & 23 & Si \\
\hline
\end{tabular}

\#/ $F_{1}$ : Familia $\underline{\text {; }} T$ : Condición laboral; $P$ : padre; $M$ : madre, $H_{1}$ : i-ésimo hijo.

entre el tiempo uno $y$ el dos, transcurrieron 5 años.

Las tasas globales de participación en la actividad laboral, de acuerdo con:

Número de personas con 12 o más años que trabajan

Número de personas con 120 más años

calculadas para los tiempos 1 y 2 son:

$$
Y_{1}=\frac{12}{19}=0.631 \quad \text { y } \quad Y_{2}=\frac{16}{22}=0.727
$$

Los subíndices de $\mathrm{Y}$ indican el momento en que se realizó la observación.

Para aplicar la ecuación (1) y examinar la composición de las tasas será necesario calcular las tasas de participación familiar y sus correspondientes ponderaciones. Los resultados se presentan en la siguiente tabla:

Tabla $N^{\circ} 2$

Tasas de participacion y ponderaciones familiares

\begin{tabular}{|c|c|c|c|c|c|c|c|c|}
\hline \multirow{3}{*}{$\begin{array}{l}\text { Tasa de } \\
\text { Part. } \\
\left(x_{j}\right)\end{array}$} & \multicolumn{4}{|c|}{ Tiempo 1} & \multicolumn{4}{|c|}{ Tiempo 2} \\
\hline & $F_{1}$ & $\mathbf{F}_{2}$ & $\mathrm{~F}_{3}$ & $P_{4}$ & $F_{1}$ & $F_{2}$ & $F_{3}$ & $F_{4}$ \\
\hline & $\frac{2}{4}=0.500$ & $\frac{2}{4}=0.500$ & $\frac{2}{3}=0.667$ & $\frac{6}{8}=0.750$ & $\frac{2}{5}=0.400$ & $\frac{3}{4}=0.750$ & $\frac{4}{6}=0.657$ & $\frac{7}{7}=1.000$ \\
\hline $\begin{array}{l}\text { Pondera } \\
\text { ción. } \\
\left(p_{i}\right)\end{array}$ & $\frac{4}{19}=0.211$ & $\frac{4}{19}=0.211$ & $\frac{3}{19}=0.421$ & $\frac{8}{19}=0.421$ & $\frac{5}{22}=0.227$ & $\frac{4}{22}=0.182$ & $\frac{6}{22}=0.273$ & $\frac{7}{22}=0.318$ \\
\hline
\end{tabular}


Sustituyendo los valores de la tabla No. 2 en la ecuación (1) tendremos para el tiempo 1:

$$
\begin{aligned}
Y_{1} & =(0.500)(0.211)+(0.500)(0.211)+(0.667)(0.158)+ \\
& +(0.750)(0.421)=0.105+0.105+0.105+0.316=0.631
\end{aligned}
$$

Y para el tiempo 2 tenemos:

$$
\begin{aligned}
Y_{2} & =(0.400)(0.227)+(0.750)(0.182)+(0.667)(0.273)+(1.000)(0.318) \\
& =0.091+0.136+0.182+0.318 \\
& =0.727
\end{aligned}
$$

Estos resultados entregan una medida de la contribución de cada familia a la formación de la tasa global. Asi la primera igualdad muestra que la cuarta familia da cuenta de la mitad; el resto se debe a los tres núcleos familiares restantes, los que realizan aportes de igual magnitud.

Cinco años después las participaciones familiares se han alterado aun cuando la cuarta familia mantiene la contribución relativa más importante $(44 \%)$. Más adelante nos preocuparemos por estudiar los cambios en las participaciones.

En el caso de las tasas bajo análisis, es conveniente percatarse de que resultan de la combinación de una determinante económica (situación laboral) y una demográfica (población de 12 o más años); mientras que las ponderaciones reconocen sólo condicionantes demográficas. En consecuencia, los cambios en las tasas de participación familiar pueden originarse en las oportunidades laborales que brinda el sistema económico o bien en la dinámica demográfica de las unidades familiares. La única forma de individualizar la fuente que produjo el cambio en la tasa es mantener los componentes de la fracción sin simplificar ni calcular su cociente.

El cambio en el aporte que realiza cada familia resulta, según la ecuación (4), del producto de las variaciones de las tasas y de los pesos. Las diferencias término a término de los componentes de $\mathrm{Y}_{1} \mathrm{y}$ $\mathrm{Y}_{2}$ son:

$$
\begin{aligned}
& Y_{2}-Y_{1}=-0.014+0.031+0.077+0.002 \\
& Y_{2}-Y_{1}=0.096
\end{aligned}
$$

La tasa de participación global se incrementó en 0.096 en los cinco años. Los valores que constituyen la diferencia nos indican que el aumento se debe a una mayor contribución de las familias 2,3 y 4 , que supera la caída experimentada por la familia 1 . 
En general, la resta término a término de la ecuación (1) evaluada en dos momentos permite apreciar el aporte que hace cada unidad (familia) al cambio de la tasa. ¿Cuáles son las fuentes de este cambio? ¿Se debe a variaciones en las tasas o en los pesos?

La ecuación (4) provee de una descomposicion a través de la cual puede construirse una respuesta a la pregunta planteada. En la tabla No 3 entregamos los cálculos básicos para computar esa ecuación:

Tabla N: 3

Incremento de las tasas y ponderaciones familiares

\begin{tabular}{ccc}
\hline Familia & Tasa $(\Delta \mathrm{x})$ & Incremento en: \\
\hline 1 & -0.100 & Ponderacion $(\Delta \mathrm{p})$ \\
2 & 0.250 & 0.017 \\
3 & 0.000 & -0.029 \\
4 & 0.250 & 0.115 \\
\hline
\end{tabular}

Con esta información y la de la tabla No 2 , sustituimos en la ecuación (4) para obtener:

$0.096=[(0.211)(-0.100)+(0.500)(0.017)+(-0.100)(0.017)]+$ $[(0.211)(0.250)+(0.500)(-0.029)+(0.250)(-0.029)]+$ $[(0.158)(0.000)+(0.667)(0.115)+(0.000)(0.115)]+$ $[(0.42 i)(0.250)+(0.750)(-0.103)+(0.250)(-0.103)]$

Realizando los productos indicados:

$$
\begin{aligned}
0.096= & {[-0.021+0.009-0.002]+[0.053-0.015-0.007] } \\
& {[0.000+0.077+0.000]+[0.105-0.077-0.026] }
\end{aligned}
$$

Cada paréntesis cuadrado corresponde a la contribución que realizan a la descomposición del cambio, las familias $1,2,3$ y 4 respectivamente. Además, se mantuvo el orden de los términos que componen la ecuación (4) de modo que el sumando:

$$
(-0.021)+(0.009)-(0.002)=-0.014
$$

descompone el aporte que realiza la primera familia a la variación en la tasa, y por lo tanto su total es igual al primer sumando de la ecuación (8). El primer término $(-0.021)$ nos dice que la cuota negativa con que concurre esta familia se debe a una caída en su tasa de parti- 
cipación, manteniendo constante su peso. La simple observación de los datos originales (ver tabla $\mathrm{N}^{\mathrm{O}}$. 1) muestra que este valor refleja un impacto del factor demográfico: sin haberse alterado el número de ocupados, la tasa desciende debido a la incorporación de un nuevo miembro a la población económicamente activa. El segundo componente (0.009) surge del cambio en la ponderación, suponiendo fija la tasa y aunque es positivo no alcanza a neutralizar el sumando anterior.

Es conveniente notar que la dinámica demográfica $\cdot$ se manifiesta de manera compleja: por una parte en el descenso de la participación, y por otra en el aumento del peso de la familia dentro del total. La desagregación que proporciona la ecuación (4) ayuda a entender e identificar las fuentes que originan estas alteraciones. La cifra -0.014 esconde los movimientos encontrados que la generan, por lo que para un análisis más detallado debe recurrirse necesariamente a (4).

El último sumando $(-0.002)$ resulta del producto de los incrementos en la tasa y en la ponderación, de ?hi cieriva su signo negativo y por su misma naturaleza su valor absoluto es pequeño. En general este tipo de términos tenderá a tomar un valor reducido por ser la resultanie del producto de dos incrementos.

Para la familia 2 tenemos:

$$
(0.053)-(0.015)-(0.007)=0.031
$$

La parte del incremento en la tasa de participación global que provoca esta familia (0.031) se debe en gran parte al aumento de su tasa familiar cuando se mantiene constante el peso (0.053). La raiz de este efecto se encuentra en la incorporación de un tercer miembro a la actividad laboral. A diferencia de 10 ocurrido en la familia anteriormente analizada, el cambio en la tasa obedece a una razón económica. La contribución positiva a $\Delta \mathrm{Y}$ se debe al predominio de este factor sobre del demográfico $(-0.015 \mathrm{y}-0.007)$.

La suma para la tercera familia es:

$$
(0.000)+(0.077)+(0.000)=0.077
$$

por lo tanto contribuye a un aumento en la tasa global y según estos sumandos se debe al cambio en la ponderación manteniendo constante la tasa (0.077). Sin embargo, este ejemplo muestra una limitación de la definición de la tasa global. En efecto, en el tiempo dos con respecto al uno, se duplicaron el número de ocupados y el de los de 12 años o más, por lo que la tasa familiar no se alteró. Nótese que en este caso se cancelan los efectos económico y demográfico y por lo 
tanto $x_{3}$ asume el valor cero. Este inconveniente también se presenta en la ecuación (4), por lo que los sumandos con valor cero no deben interpretarse como ausencia de modificaciones. Para una justa evaluación de este resultado habrá que examinar los componentes de la tasa familiar con base en los valores originales.

En la última familia tenemos:

$$
(0.105)-(0.077)-(0.026)=0.002
$$

Estas cifras expresan que la cuarta unidad familiar contribuye levemente al aumento de la tasa global de participación. El incremento en la tasa faniliar con ponderación constante $(0.105)$ es superior a la disminución que se produce por la caída del peso $(-0.077 \mathrm{y}-0.026)$. El alza en el valor de $x_{4}$ se debe a que se enfatizó la participación laboral de la familia (ingresan dos miembros y sale uno) reforzada por una disminución de la población económicamente activa de esa familia. Este mismo hecho explica la baja de la ponderación que origina el signo negativo de los otros dos sumandos.

La aplicación de la ecuación (1) ayudó a examinar el impacto de las tasas familiares de participación sobre la global. Su uso en dos puntos del tiempo hizo posible determinar la contribución de cada familia a $\Delta \mathrm{Y}$ y a través de la ecuación (4) se identificaron los factores que operan sobre las modificaciones de los aportes familiares a través del tiempo.

Con el propósito de ilustrar el uso de las ecuaciones (6) y (7) referidas a grupos de observaciones, supongamos que se forma una clase (que denominaremos A) por la agrupación de las familias 1 y 2 , mientras que las dos restantes conforman otra clase que designaremos por B. Al agregar la información contenida en la tabla No 1 y proceder al cálculo de las tasas y ponderaciones correspondientes a las clases llegamos a:

Trbla No 4

Tasas de participación y ponderaciones por clase

\begin{tabular}{lllll} 
& Clage A & Tiampo 1 & & Tlempo 2 \\
Tasa de Part. $\left(\mathrm{x}_{\mathrm{h}}\right)$ & $\frac{4}{8}=0.500$ & $\frac{8}{11}=0.727$ & $\frac{5}{9}=0.556$ & $\frac{11}{13}=0.846$ \\
Porderación $\left(P_{h}\right)$ & $\frac{8}{19}=0.421$ & $\frac{11}{19}=0.579$ & $\frac{9}{22}=0.409$ & $\frac{13}{22}=0.591$ \\
\hline
\end{tabular}


Para determinar las contribuciones de los grupos a la formación de la tasa global alimentaremos la ecuación (6) con las cifras de esta tabla:

$$
\begin{aligned}
Y_{1} & =(0.500)(0.421)+(0.727)(0.579) \\
& =0.211+0.420 \\
& =0.631
\end{aligned}
$$

En consecuencia podemos afirmar que la clase B reatiza un aporte a la tasa de participación laboral del doble que la $\mathrm{A}$. Para el segundo instante tenemos:

$$
\begin{aligned}
Y_{2} & =(0.556)(0.409)+(0.846)(0.591) \\
& =0.227+0.500 \\
& =0.727
\end{aligned}
$$

Estos valores indican que la cuota del agregado B sigue siendo sustancialmente mayor que la del A.

La diferencia término a término $\left(\mathrm{Y}_{2}-\mathrm{Y}_{1}\right)$ muestra la forma como se integra la variación de la tasa global:

$$
\begin{aligned}
Y_{2}-Y_{1} & =(0.227-0.211)+(0.500-0.420) \\
& =0.016+0.080 \\
& =0.096
\end{aligned}
$$

La mayor parte del aumento se explica por el cambio experimentado por la clase B. Si fuera necesario identificar el impacto de los factores económico y demográfico, deberíamos llevar a cabo un análisis de las variaciones en las tasas y ponderaciones tal como se hizo para las unidades familiares.

Sin embargo, la descomposición que nos entrega la ecuación (7) ayuda a identificar si la contribución de cada clase se debe a un efecto tasa, a un efecto ponderación o a la combinación de ambos.

Para el cálculo numérico de la ecuación (7) se requieren las variaciones en las tasas y pesos.

Tabła No 5

\begin{tabular}{lcc}
\hline Clase & Tasa $(\Delta x)$ & Incremento en: \\
\hline A & 0.056 & Ponderacion $(\Delta \mathrm{P})$ \\
B & 0.119 & -0.012 \\
\hline
\end{tabular}


Estos datos y los contenidos en la tabla No 4 al sustituirse convenientemente en (7) arrojan por resultado:

$$
\begin{aligned}
\Delta Y & =[(0.421)(0.056)-(0.500)(0.012)-(0.056)(0.012)]+ \\
& +[(0.579)(0.119)+(0.727)(0.012)+(0.119)(0.012)] \\
& =[(0.025-0.006-0.002]+[0.069+0.009+0.001] \\
& =0.017+0.079 \\
& =0.096
\end{aligned}
$$

Esta información indica que el aumento que experimentó el agregado $\mathrm{A}$ en el periodo bajo consideración se origina fundamentalmente en un cambio en la tasa de participación de la clase manteniendo fija la ponderación (0.025). Esta cifra contrarresta los impactos a la baja que inducen el cambio en la ponderación bajo el supuesto de que la tasa de participación de la clase se mantiene constante, y el de las variaciones conjuntas de los pesos y las tasas.

Sabemos que la contribución más importante a $\Delta Y$ la hace el grupo $B$. Los valores numéricos que se encuentran dentro del segundo paréntesis cuadrado de la igualdad anterior nos dicen que una parte sustancial de dicho aporte (0.069) se debe al aumento en la tasa de la clase cuando se fija el valor de la ponderación. La influencia de esta última actúa en el mismo sentido aun cuando tiene una importancia relativamente marginal (0.009). La combinación de las variaciones en tasa y peso es prácticamente nula (0.001).

Hasta el momento hemos presentado dos tipos de desarrollos numéricos independientes. Uno en que las unidades fueron las familias $\mathrm{y}$ otro en que fueron agregados de familias. El vínculo entre ambos niveles del análisis está dado por las ecuaciones (5) que se presentaron en la sección anterior. En ella se estableció que si la contribución del agregado podía expresarse como una combinación lineal de los aportes de las unidades que lo conforman, entonces queda a nuestra disposición un instrumento que permite estudiar el impacto de las observaciones individuales sobre la tasa del grupo y sobre su cambio en el tiempo.

Veamos a manera de ejemplo la validez de (5) para el caso de la clase A:

$\begin{aligned} \frac{(T R A B \geqslant 12) A}{(P . E . A .) A} \cdot \frac{(P . E . A .) A}{\text { P.E.A. }} & =\frac{(T R A B \geqslant 12) A, 1}{(P . E . A .) A, 1} \cdot \frac{(P . E . A .) A, 1}{(\text { P.E.A. } A} \cdot \frac{(\text { P.E.A. } A}{\text { P.E.A. }}+\ldots \cdots+ \\ & +\frac{(T R A B \geqslant 12) A, n_{A}}{\left(\text { P.E.A.) } A, n_{A}\right.} \cdot \frac{\left(\text { P.E.A.) } A, n_{A}\right.}{\text { (P.E.A. } A} \cdot \frac{\text { (P.E.A. } A}{\text { P.E.A. }}\end{aligned}$


Al eliminar los términos indicados:

$$
\begin{aligned}
\frac{(T R A B \geqslant 12) A}{(P . E . A .) A} \quad \frac{(P . E . A .) A}{(P . E . A .)} & =\frac{(T R A B \geqslant 12) A .1}{(P . E . A .) A, 1} \cdot \frac{(\text { P.E.A. }) A .1}{(\text { P.E.A. })}+\ldots \ldots+ \\
& +\frac{(\text { TRAB } \geqslant 12) A, n_{A}}{(\text { P.E.A. }) A, n_{A}} \frac{(\text { P.E.A. }) A, n_{A}}{(\text { P.E.A. })}
\end{aligned}
$$

En esta igualdad se reconocen los elementos que intervienen en las ecuaciones (5). En efecto:

$\frac{(T R A B \geqslant 12) A}{(\text { P.E.A. }) A}=X_{A}$; es la tasa de participación del grupo, por cuanto establece la relación entre el número de trabajadores en el grupo A y sus miembros pertenecientes a la población económicamente activa.

$\frac{\text { (P.E.A.) A }}{\text { P.E.A }}=\mathrm{P}_{\mathrm{A}}$; denota el peso de la clase A dentro de la población económicamente activa global.

Los términos del lado derecho de la ecuación fueron empleados con anterioridad pero con diferente simbología. Los subíndices $A, 1$; $\ldots \ldots ; \mathrm{A}, \mathrm{n}_{\mathrm{A}}$ se refieren a la primera, ....., n-ésima familia dentro del grupo A. Por consiguiente, al sustituir tendremos.

$$
\begin{aligned}
& X_{A} P_{A}=x_{A, 1} p_{A, 1}+X_{A, 2} p_{A, 2}+\ldots \ldots \ldots+x_{A}, n_{A} p_{A}, n_{A} \\
& X_{A} P_{A} \stackrel{n_{A}}{=\sum} x_{A}, i p_{A}, i
\end{aligned}
$$

Como las clases A y B se definieron de la misma manera, entonces el hecho de que (5) sean válidas para A permite extender este resultado a B.

Con los datos del ejemplo numérico desarrollado en esta sección mostraremos que las ecuaciones (5) ligan la contribución de cada grupo a la tasa global con los aportes que realizan las unidades que 1o conforman.

Sabemos que:

$$
X_{\mathrm{A}} P_{\mathrm{A}}=\frac{4}{19}=0.211
$$


como la clase A incluye a las dó primeras familias y sus tasas de participación y ponderaciones en el tiempo 1 son, según la tabla No 2:

$\mathrm{x}_{\mathrm{A}, 1}=0.500 \quad \mathrm{p}_{\mathrm{A}, 1}=0.211 \quad \mathrm{x}_{\mathrm{A}, 2}=0.500 \quad \mathrm{p}_{\mathrm{A}, 2}+0.211$

entonces se cumple que:

(10) $0.211=(0.500)(0.211)+(0.500)(0.211)$

y en la clase B, también en el tiempo 1:

$$
\begin{aligned}
& \mathrm{x}_{\mathrm{B}} \mathrm{p}_{\mathrm{B}}=\frac{8}{19}=0.420 \quad \mathrm{y} \\
& \mathrm{x}_{\mathrm{B}, 1}=0.667 \quad \mathrm{p}_{\mathrm{B}, 1}=0.158 \quad \mathrm{x}_{\mathrm{B}, 2}=0.750 \quad \mathrm{p}_{\mathrm{B}, 2}=0.421
\end{aligned}
$$

de manera que:

$$
0.420=(0.667)(0.158)+(0.750)(0.421)
$$

Cuando se satisfacen las condiciones impuestas por las ecuaciones (5) resulta sencillo obtener la importancia de los agregados en el valor de la tasa de participación. Para ello bastará con recurrir a la igualdad (2); una vez conocida la composición familiar de los grupos se suman las contribuciones de cada unidad. Si recordamos que:

$$
\begin{array}{r}
Y_{1}=[(0.500)(0.211)+(0.500)(0.211)]+[(0.667)(0.158)+ \\
(0.750)(0.421)]
\end{array}
$$

da la composición de la tasa en el tiempo 1 en función de las familias y tomando en cuenta la definición de los agregados, se pueden obtener sus contribuciones mediante la adición de los términos correspondientes. En este caso los paréntesis cuadrados muestran las cuotas de las clases, lo que se corrobora al compararlos con los resultados de (10) y (11).

Este hecho es de suma importancia operativa porque hace posible examinar el impacto de los agregados en el cambio y su composición, a través de las ecuaciones correspondientes a las familias. La diferencia entre las tasas (véase la igualdad (3)), analizada desde el punto de vista de los grupos será:

$$
\begin{aligned}
Y_{2}-Y_{1} & =0.017+0.079 \\
& =0.096
\end{aligned}
$$


en esta ecuación se constata que el aumento en la tasa de participación laboral proviene básicamente de la clase $\mathrm{B}$.

De manera análoga, la composición del cambio al nivel agregado resulta de la suma algebraica de los elementos correspondientes de (9):

$$
\begin{aligned}
Y_{2}-Y_{1} & =[0.032-0.006-0.009]+[0.105+0.000-0.026] \\
& =0.017+0.079 \\
& =0.096
\end{aligned}
$$

La interpretación de los componentes de esta diferencia es similar a la que ya hemos presentado por lo que no creemos necesario repetirla.

Si las ecuaciones del modelo se calculan para unidades familiares y además se definen agregados de familias, será posible analizar el impacto de los grupos sobre la composición y el cambio en la tasa global de participación laboral por la suma de las contribuciones individuales. Lo inverso no es válido ya que resultará imposible moverse desde los resultados agregados hacia las unidades familiares que los integran.

\section{Conclusiones}

En la segunda sección presentamos un conjunto de ecuaciones para analizar la composición de una tasa susceptible de expresarse como combinación lineal, examinar su cambio en el tiempo y los vínculos que ligan sus distintos niveles de agregación.

La tercera sección se dedicó a una aplicación de dichas ecuaciones a un conjunto hipotético y reducido de familias para las cuales se calcularon sus tasas de participación en la actividad laboral. El hecho de que trabajásemos con cuatro familias, dos clases y dos momentos en el tiempo corresponde a restricciones operativas relacionadas con el manejo del ejemplo. La estructura del modelo le otorga validez general en lo que se refiere al número de casos, la cantidad de agregados y los tiempos considerados.

Es posible generalizar las ecuaciones de agregación no sólo a varias clases sino también a diferentes niveles de agrupación. Si se cumple la condición de que las clases se forman por combinación lineal de las subclases y éstas a su vez por combinación lineal de las sub-subclases $\mathrm{y}$ así sucesivamente hasta llegar a las observaciones originales, entonces será posible analizar las tasas y sus cambios en cada nivel, por acumulación de los niveles inferiores. 
La extensión de estos resultados también abarca lo temporal. En efecto, se puede demostrar que las variaciones son adicionales en el tiempo, de manera que si al cambio entre los momentos uno y dos le sumamos el correspondiente al dos y tres, obtendremos como resultado el que ocurrió entre uno y tres.

La naturaleza del modelo indica que si estamos interesados en llevar a cabo un análisis dinámico, será necesario seguir la trayectoria en el tiempo del conjunto inicial de observaciones. Esto reduce su aplicabilidad a información proveniente de paneles. No hemos considerado en este trabajo los procesos que afectan al número de observaciones, este tema tendrá que ser la preocupación principal de los futuros desarrollos en esta línea. 